\title{
Mechanisms underlying the formation and enlargement of noncommunicating syringomyelia: experimental studies
}

\author{
Marcus A. Stoodley, Ph.D., F.R.A.C.S., Nigel R. Jones, D.Phil., F.R.A.C.S, \\ LiQun YANG, M.D., AND Christopher J. BROWN, B.APP.SC. \\ Institute of Neurological Sciences, Prince of Wales Hospital, University of New South Wales, \\ Randwick, New South Wales; and Department of Surgery (Neurosurgery), University of Adelaide, \\ Adelaide, South Australia
}

\begin{abstract}
The pathogenesis of noncommunicating syringomyelia is unknown, and none of the existing theories adequately explains the production of cysts that occur in association with conditions other than Chiari malformation. The authors hypothesis is that an arterial pulsation-driven perivascular flow of cerebrospinal fluid (CSF) is responsible for syrinx formation and enlargement. They investigated normal CSF flow patterns in 20 rats and five sheep by using the tracer horseradish peroxidase; the effect of reducing arterial pulse pressure was examined in four sheep by partially ligating the brachiocephalic trunk; CSF flow was examined in 78 rats with the intraparenchymal kaolin model of noncommuthe brachiocephalic trunk; CSF flow was examined in 78 rats with the intraparenchymal kaolin model of non
nicating syringomyelia; and extracanalicular cysts were examined using the excitotoxic model in 38 rats.

nicating syringomyelia; and extracanalicular cysts were examined using the excitotoxic model in 38 rats.
In the normal animals there was a rapid flow of CSF from the spinal subarachnoid space into the spinal cord perivascular spaces and then into the central canal. This flow ceased when arterial pulsations were diminished. In animals with noncommunicating syringomyelia, there was rapid CSF flow into isolated and enlarged segments of central canal, even when these cysts were causing pressure damage to the surrounding spinal cord. Exitotoxic injury of the spinal cord caused the formation of extracanalicular cysts, and larger cysts were produced when this injury was combined with arachnoiditis, which impaired subarachnoid CSF flow. The results of these experiments support the hypothesis that arterial pulsation-driven perivascular fluid flow is responsible for syrinx formation and enlargement.
\end{abstract}

KEY WORDS • syringomyelia • cerebrospinal fluid • spinal cord • perivascular space $\bullet$ central canal

Cysts develop in the spinal cord in association with many congenital and acquired disorders, including spina bifida, hindbrain abnormalities, neoplasms, trauma, meningitis, and arachnoiditis. In patients with spina bifida all cerebral ventricles are generally enlarged and a dilated central canal is found in continuity with the fourth ventricle. ${ }^{8}$ In such cases the probable mechanism of syrinx formation and enlargement is transmission of arterial and venous pressure waves from the ventricles into the central canal via the fourth ventricle, which has occluded foramina, or possibly by a valve mechanism of the herniated cerebellar tonsils. ${ }^{4,20} \mathrm{~A}$ ventricular CSF diversion procedure to control the hydrocephalus will generally be effective treatment for this communicating type of syringomyelia.

Early theories of syrinx formation were based on the assumption that there was a communication between the syrinx and the fourth ventricle in all cases. ${ }^{4,20}$ However, magnetic resonance imaging, pathological and intraopera-

Abbreviations used in this paper: $\mathrm{CSF}=$ cerebrospinal fluid; GFAP = glial fibrillary acidic protein; HRP = horseradish peroxidase; $\mathrm{QA}=$ quisqualic acid. tive studies have demonstrated that the majority of spinal cord cysts do not communicate with the fourth ventricle. ${ }^{6-9,13}$ Noncommunicating syringomyelia can result from enlargement of an isolated segment of the central canal ("canalicular syringomyelia") or from a cyst that forms primarily in the spinal cord parenchyma ("extracanalicular syringomyelia"). Canalicular syringomyelia occurs in association with hindbrain abnormalities, and neurological deficits associated with this type of cyst occur when the syrinx ruptures into the spinal cord parenchyma. ${ }^{8}$ Extracanalicular syringomyelia occurs after trauma and in association with tumors of the spinal cord, and neurological deficits, in these cases, are related to the spinal cord region damaged by the cyst.

Noncommunicating syringomyelia is therefore a highly heterogeneous condition. Its pathogenesis is unknown; indeed, it is even unclear whether a common mechanism is responsible for cysts associated with different conditions. In the past authors generally attempted to explain the formation and enlargement of only those cysts associated with Chiari malformations. ${ }^{1,4,12,20}$ None of the existing hypotheses, however, satisfactorily explains the formation of syringomyelia associated with other conditions. 
In our hypothesis we posit that CSF normally flows from the spinal subarachnoid space into the central canal via the perivascular spaces and that syringomyelia occurs when various conditions perturb this inflow or the egress of fluid from the central canal. We performed a series of experiments to investigate this hypothesis.

\section{MATERIAL AND METHODS}

Ethics approval was obtained for all experiments from the Ethics Committees of the University of Adelaide and the Institute of Medical and Veterinary Science. The animals used in these experiments were handled in a humane fashion, and the experimental procedures complied with the Code of Practice of the Australian National Health and Medical Research Council. The experimental procedures have been detailed previously; ${ }^{15-17}$ an abbreviated account will follow.

All procedures were performed in the experimental animals after induction of inhalational general anesthesic of isoflurane and oxygen. Sheep were intubated and mechanically ventilated, and rats were allowed to self-ventilate through a nose cone. Horseradish peroxidase (Zymed Laboratories, San Francisco, CA) was used as the CSF tracer in all experiments; a concentration of 3\% HRP in normal saline was injected into the spinal subarachnoid space or the cisterna magna. The tracer was localized in $50-\mu$ vibratome sections of brain and spinal cord by using the chromagen tetramethylbenzidine stabilized with ammonium molybdate. All animals were perfuse fixed with paraformaldehyde in phosphate buffer. The spinal cord and brain were post fixed in paraformaldehyde. The entire cervical special cord was studied in detail in each animal, and sections from the thoracic and lumbar spinal cord were also examined.

\section{Experimental Animal Groups}

Normal Fluid Flow. Normal fluid flow in the spinal cord was examined in 20 male Sprague-Dawley rats and five wether merino sheep. One group of 10 rats received tracer injection into the cisterna magna. In a second group of 10 rats tracer was injected into the thoracic spinal subarachnoid space to avoid the possibility of the material entering the central canal via the fourth ventricle. Tracer was injected into the cervical subarachnoid space in the sheep. In one control animal in each group, normal saline without HRP was injected into the subarachnoid space. The sheep were killed by rapid perfusion fixation at $10 \mathrm{~min}$ utes post-CSF tracer injection, and the rats were examined immediately after injection, 10 minutes postinjection or 30 minutes postinjection (three rats at each time in each group).

Fluid Flow With Reduced Pulse Pressure. Four sheep were used to examine the effect of reducing arterial pulse pressure. The brachiocephalic trunk was partially ligated while monitoring the pulse pressure so that the pulse pressure was reduced with minimal alteration of mean arteria pressure. The CSF tracer was then injected and the animals studied as with the normal group. In one control animal a sham ligation was performed; the methods of tracer injection and tissue processing were otherwise identical to those in the remainder of the group.
Fluid Flow in an Animal Model of Canalicular Syringomyelia. The intraparenchymal kaolin model of noncommunicating syringomyelia was used in 78 rats. The animals were divided into four groups according to the time after kaolin injection at which the CSF flow was studied (1 day, 3 days, 1 week, and 6 weeks postinjection). Each group was subdivided into three groups that were studied immediately after HRP injection, at 10 minutes postinjection, or at 30 minutes postinjection. Cerebrospinal fluid flow was studied using HRP tracer injected either in the cisterna magna or the thoracic subarachnoid space. Sections were acquired at each spinal cord level for analysis of fluid flow, and the remainder of the cervical spinal cord was sectioned longitudinally or transversely and stained with hematoxylin and eosin. The entire cervical spinal cord was studied histologically to identify isolated and dilated segments of central canal and to make correlations between fluid flow and central canal morphological findings.

Animal Model of Extracanalicular Syringomyelia. This group consisted of 38 rats divided into five subgroups. In a control group (Group A, [three rats]) $2 \mu \mathrm{L}$ normal saline containing $0.2 \%$ Evans Blue was injected into the dorsal columns of the lower cervical spinal cord; in Group B (five rats) $5 \mu \mathrm{L}$ normal saline containing $250 \mathrm{mg} / \mathrm{mL}$ kaolin suspension was injected into the cervical spinal subarachnoid space; in Group C (10 rats) $2 \mu \mathrm{L}$ normal saline containing $1.6 \mathrm{mg} / \mathrm{mL}$ QA (Sigma Chemical Corp, St. Louis, MO) was injected into the lower cervical cord dorsal columns; in Group D (nine rats) a higher concentration of QA ( $2 \mu \mathrm{L}$ of $23.7 \mathrm{mg} / \mathrm{mL}$ ) was injected into the cervical cord; and in Group E (11 rats) high-concentration QA was injected into the cervical cord followed by an injection of $5 \mu \mathrm{L}$ of kaolin $250 \mathrm{mg} / \mathrm{mL}$ into the cervical subarachnoid space. The animals were killed by perfusion fixation after induction of anesthesia at 1 to 4 weeks postinjection. Transverse cervical spinal cord sections were cut for staining with hematoxylin and eosin and for immunocytochemical analysis for GFAP (Dako Corp, Carpinteria, CA) and for the macrophage marker ED1, a mouse anti-rat monoclonal antibody (Serotec Ltd., Oxford, UK). One normal rat was used as a control for immunocytochemical and morphological features of the cervical spine.

\section{RESULTS}

\section{Normal Fluid Flow}

Horseradish peroxidase was detectable in the pia and underlying extracellular space at all levels of the spinal cord immediately after cisternal injection in rats; the transpial depth of tracer staining was proportional to the distance from the injection point. There was rapid flow of CSF from the subarachnoid space into perivascular spaces throughout the spinal cord and particularly in the central grey matter (Fig. 1). Although CSF had not reached the most rostral levels of the spinal cord immediately after the stain was injected into the thoracic subarachnoid space, by 10 minutes postinjection there was evidence of CSF flow into the perivascular spaces at all levels of the spinal cord.

In the animals killed immediately after either cisternal or thoracic injection and in the group studied at $10 \mathrm{~min}-$ 


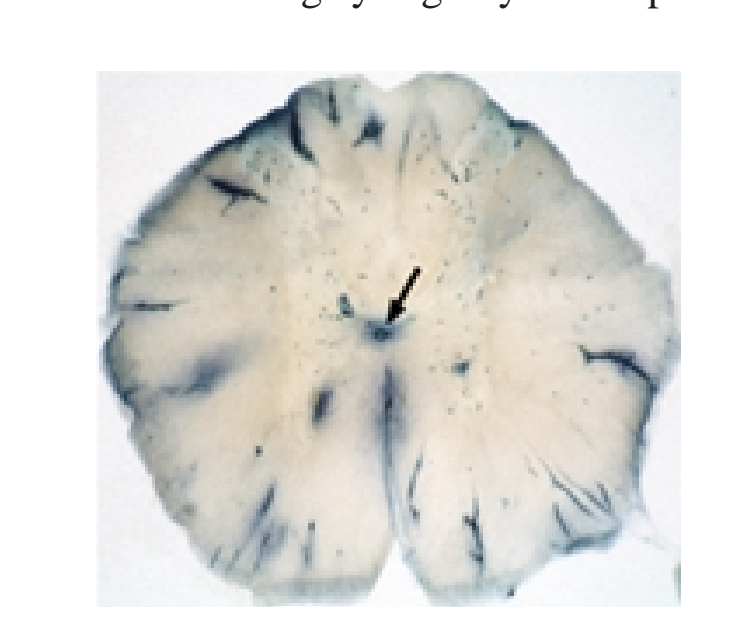

Fig. 1. Photomicrograph of a transverse section of a rat thoracic spinal cord obtained 10 minutes after injecting HRP into the cisterna magna. Blue HRP reaction product is present in numerous perivascular spaces and has already reached the central canal ( $a r-$ row). Original magnification $\times 25$.

utes postinjection, there was evidence of a rapid fluid flow from the central grey matter perivascular spaces into the central canal via the extracellular spaces and between ependymal cells (Fig. 2). This flow was always directed preferentially toward the central canal rather than spreading circumferentially away from perivascular spaces. By 30 minutes postinjection widespread labeling of the extracellular spaces was demonstrated in the central grey matter, and patterns of flow could not be determined.

Similar patterns of flow were demonstrated in the sheep group. By 10 minutes postinjection HRP was detectable at each level of the cervical cord; the thoracic and lumbar levels were not labeled at this time. Perivascular spaces were labeled throughout the cervical spinal cord, predominantly at the level of injection. The pattern of HRP staining indicated a preferential flow from the perivascular spaces into the central canal (Fig. 3).

\section{Influence of Pulse Pressure}

There were no changes in arterial, intracranial, or intraspinal pressure in the animal undergoing sham brachioce-

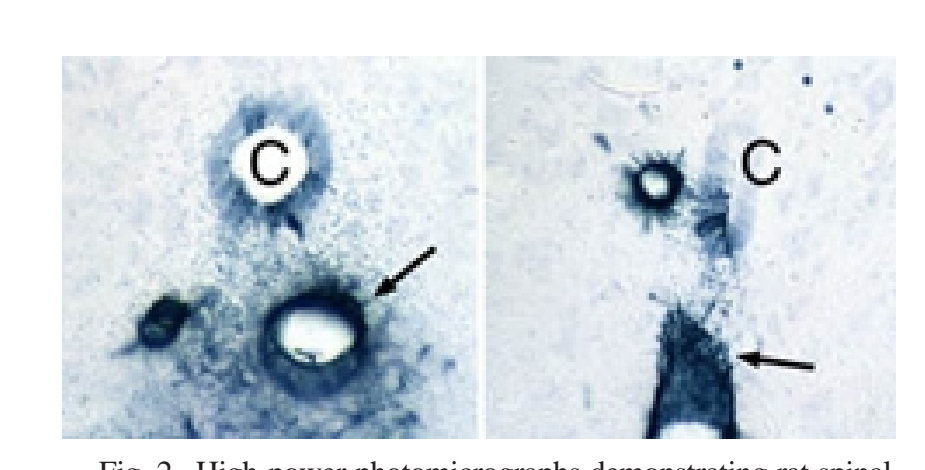

Fig. 2. High-power photomicrographs demonstrating rat spinal cord central grey matter after injection of HRP into the cisterna magna. Left: Lumbar spinal cord 10 minutes postinjection. Right: Conus medullaris immediately postinjection. The pattern of HRP Conus medullaris immediately postinjection. The pattern of HRP
reaction product indicates a flow of CSF from the perivascular spaces (arrows) towards the central canal (C). Original magnification $\times 200$

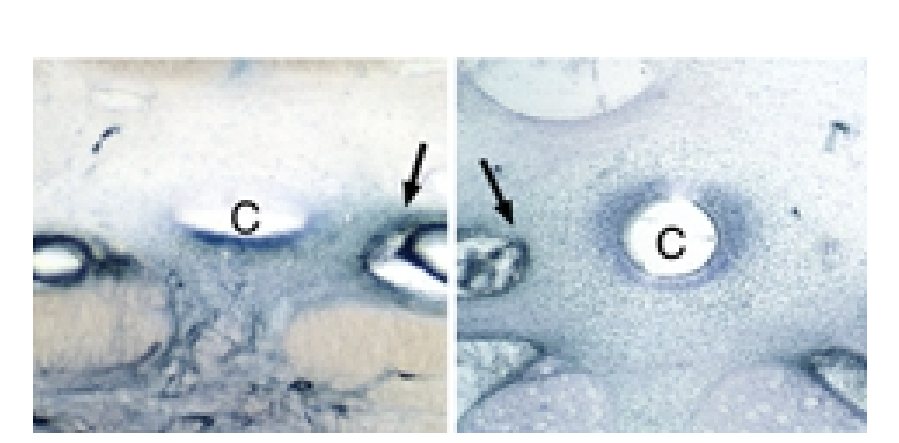

Fig. 3. Photomicrographs of transverse sections of sheep cervical spinal cord obtained 10 minutes after HRP injection into the cervical subarachnoid space. The distribution of reaction product is suggestive of a flow of CSF from central grey matter perivascular spaces (arrows) towards the central canal. Original magnification $\times 100$.

phalic ligation, and in this animal the patterns of CSF flow were similar to those demonstrated in the normal sheep, with a preferential flow of fluid from perivascular spaces into the central canal (Fig. 4). There was dampening of the arterial, intracranial, and intraspinal pulse pressures in the animals in which brachiocephalic ligation was performed. The mean pressures were not substantially lower postligation as compared with preligation. The CSF tracer was detectable in perivascular spaces in the superficial white matter but not in the central grey matter, and there was no evidence of flow into the central canal, which did not contain tracer (Fig. 4).

\section{Flow of CSF in Canalicular Syringomyelia}

The central canal was occluded at the level at which the kaolin was injected and at one or more rostral levels in each animal (Fig. 5). The segments of central canal that were isolated between occlusions gradually dilated (Fig. 6), and there was evidence of pressure damage (axonal retraction balls and edema) in the surrounding spinal cord. Perivascular spaces became dilated. In this group of animals, CSF did not flow in the subarachnoid space as rapidly as in the normal animals; at the level at which kaolin was injected there was a partial blockage of flow, both in the animals that received a cisternal and thoracic tracer injection. The extent of blockage resolved over time, with a more rapid dispersion of HRP throughout the neuraxis in the 6-week postinjection group than in those studied at earlier time points.

At all stages of syrinx development there was rapid flow of CSF tracer from the perivascular spaces into the central canal (Fig. 7). This occurred when the central canal was isolated by occlusions at each end and became dilated, at spinal cord levels where the canal was filled with kaolin or inflammatory cells, and in segments where there was evidence of pressure damage caused by the enlarged central canal on the surrounding spinal cord.

\section{Animal Model of Extracanalicular Syringomyelia}

Transient unilateral forelimb weakness, which resolved within 3 days, was observed in many of the rats that had received injections into the spinal cord. No subsequent neurological deficits occurred in any of the groups. A mild inflammatory response and positive GFAP staining were 


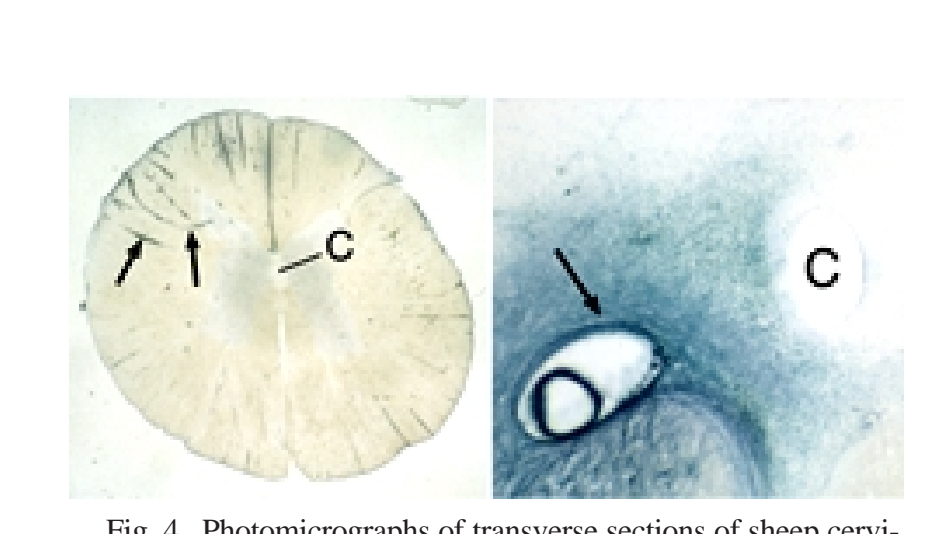

Fig. 4. Photomicrographs of transverse sections of sheep cervisubarachnid space. Left: Section obtained in an animat undergosubarachnoid space. Left: Section obtained in an animal undergoing partial ligation of the brachiocephalic trunk to reduce pulse pressure. There is HRP staining in superficial perivascular spaces (arrows) but no evidence that it reached the central grey matter or central canal. Original magnification $\times 15$. Right: Section obtained in an animal undergoing sham ligation of the brachiocephalic trunk without alteration of pulse pressures. There is heavy staining of a central grey matter perivascular space and evidence of CSF flow from the perivascular space (arrow) to the cenitral can of Original magnification $\times 100$

observed in the group that received normal saline injection; no spinal cord cysts were demonstrated in this group and the central canal remained normal. In the group that received subarachnoid kaolin injections arachnoiditis developed in the subarachnoid space, which contained kaolin crystals and inflammatory cells. No cell loss, cystic change, or other abnormality was observed in the grey or white matter, and the central canal remained normal.

Eight of 10 rats in the low-concentration QA group developed spinal cord cysts. The small, well-defined cysts did not communicate with the central canal. We observed intense GFAP-stained hypertrophic astrocytes near the cyst wall, and ED1-positive macrophages were scattered throughout the parenchyma. A more marked inflammatory response was observed in the high-concentration QA group in which eight of the nine rats developed cysts. Compared with the low-concentration group, the cysts in this group were larger and extended over multiple segments. The cysts did not communicate with the central canal, which remained normal. The largest cysts were demonstrated in the group that had received high-concentration QA and subarachnoid kaolin injections. Nine of the 11 rats in this latter group developed cysts, all of which exceeded two spinal cord segments in length (Fig. 8). The cysts in this group were often multiple, and there was an extensive inflammatory response and astrocytic reaction in the parenchyma. There was circumferential arachnoiditis, which appeared to obstruct the subarachnoid space, at the level where the kaolin was injected.

\section{DISCUSSION}

It has been assumed, although not proven, that the fluid in syrinx cavities is CSF that has entered the cyst from either the ventricles or subarachnoid space. Early hydrodynamic theories of syringomyelia pathogenesis were predicated on the assumption of a communication, in all cases, between the fourth ventricle and the syrinx. The
M. A. Stoodley, et al.

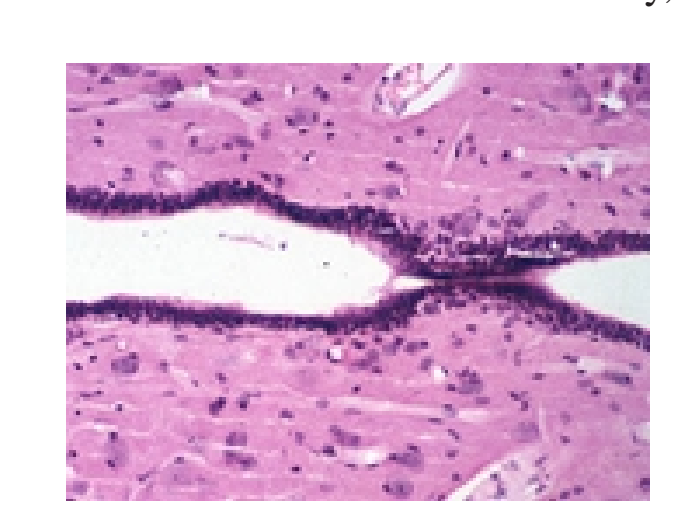

Fig. 5. Photomicrograph of a longitudinal section of rat cervical spinal cord obtained 1 week after intraparenchymal kaolin injection. The central canal is blocked by an adhesion and it is dilated on both sides of the adhesion. $\mathrm{H} \& \mathrm{E}$, original magnification $\times$ 200.

focus was therefore on explanations of pressure transmission from the intracranial space into the central canal via the fourth ventricle, either as a direct transmission of pulsations ("water hammer" effect) or as a craniospinal pressure differential that results from a supposed valve effect of the herniated cerebellar tonsils., ${ }^{4,21}$ These theories might have some merit in cases in which a fourth ventricle-to-syrinx communication does exist; however, it has been demonstrated that the majority of syringes do not have a direct connection with the fourth ventricle. ${ }^{6-9,13}$ In addition, these theories do not explain the formation of cysts associated with conditions other than Chiari malformations.

Ball and Dayan ${ }^{1}$ and Nogués ${ }^{11}$ have suggested that the entry route for CSF might be the perivascular spaces or dorsal root entry zone and that the impetus for fluid inflow was intracranial pulsations transmitted by the cerebellar tonsils that are impacted in the foramen magnum. This general theory has been championed further by Oldfield and colleagues, 5,12 who have suggested that syrinx formation is caused by the cerebellar tonsils acting as a piston to produce large pressure waves in the spinal subarachnoid space, and this action forces fluid through the surface of the spinal cord or the perivascular spaces into the central canal. Once formed, such syringes are believed to enlarge due to external compression of the cord, which increases the pressure in the syrinx and causes longitudinal dissection of the spinal cord. This is essentially identical to the "slosh" theory proposed by Williams, ${ }^{21}$ who has argued that the external force on the spinal cord is caused by the raised intraspinal pressure due to maneuvers such as coughing and straining.

Although intermittent compression of existing cysts could conceivably account for syrinx enlargement by causing longitudinal dissection in the spinal cord, it is difficult to imagine that forces acting on the cord surface could be responsible for enlarging the microscopic central canal to form a cyst. Furthermore, all of these theories propose the existance of a mechanism that would only apply to cysts associated with Chiari malformations and that cannot explain canalicular syringomyelia due to other causes or extracanalicular syringomyelia due to any cause. We believe that the formation and enlargement of noncommunicating syringes are likely to share a common 


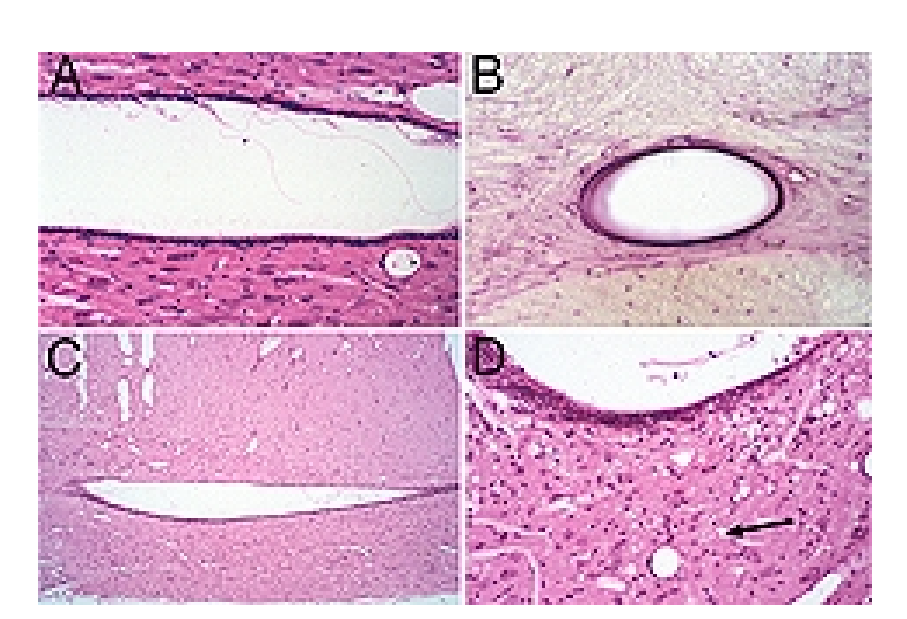

Fig. 6. Photomicrographs demonstrating the rat intraparenchymal kaolin model of noncommunicating syringomyelia. After injection of kaolin into the spinal cord dorsal columns, segments of central canal become isolated and progressively dilated. A Longitudinal section of cervical spinal cord obtained 1 week postinjection, showing a dilated central canal that was isolated at each end by occlusions of the canal. B: Cervical spinal cord cross section obtained 3 days postinjection, demonstrating a dilated centra canal. C: Low-power longitudinal section of upper cervical spinal cant. C: Low-por an massively dilated. D: Transverse section of cervical cord obtained 6 weeks postinjection; there is interstitial edema and axonal retraction balls (arrow) provide evidence of tissue damage from the enlarged central canal. $\mathrm{H} \& \mathrm{E}$, original magnification $\times 40(\mathrm{C})$ and $\times 200(\mathrm{~A}, \mathrm{~B}$, and $\mathrm{D})$.

pathophysiological mechanism regardless of the associated condition. Our hypothesis is that CSF flows normally along the perivascular spaces from the spinal subarachnoid space and that perturbations of this flow caused by Chiari malformations or other conditions, are responsible for cyst formation and enlargement.

\section{Normal Fluid Flow in the Spinal Cord}

Flow of CSF from the subarachnoid space into perivascular spaces has been demonstrated by many investigators following the work by Weed ${ }^{19}$ and Cushing ${ }^{3}$ in 1914 Rennels and coworkers ${ }^{14}$ have provided evidence that CSF rapidly circulates from the subarachnoid space into the perivascular spaces of the brain and spinal cord. In addition to supporting this concept, our findings in rats and sheep suggest that there is a preferential flow of CSF from the perivascular spaces into the central canal. There is an anatomical basis for such a flow because the central canal is surrounded by labyrinthine structures formed by basement membranes that are associated with subependymal capillaries. These central grey matter labyrinths communicate with the perivascular spaces of arteries entering the central grey matter. ${ }^{2}$

Presumably there is a driving force for a rapid perivascular fluid flow. Rennels and coworkers ${ }^{14}$ have demonstrated that the flow did not occur when arterial pulse pressures were reduced in dogs, and they suggested that arterial pulsations were responsible for propelling perivascular fluid. Our results with a similar experiment in sheep support this theory; when the pulse pressure was reduced with minimal alteration of mean pressure, there was a dra-

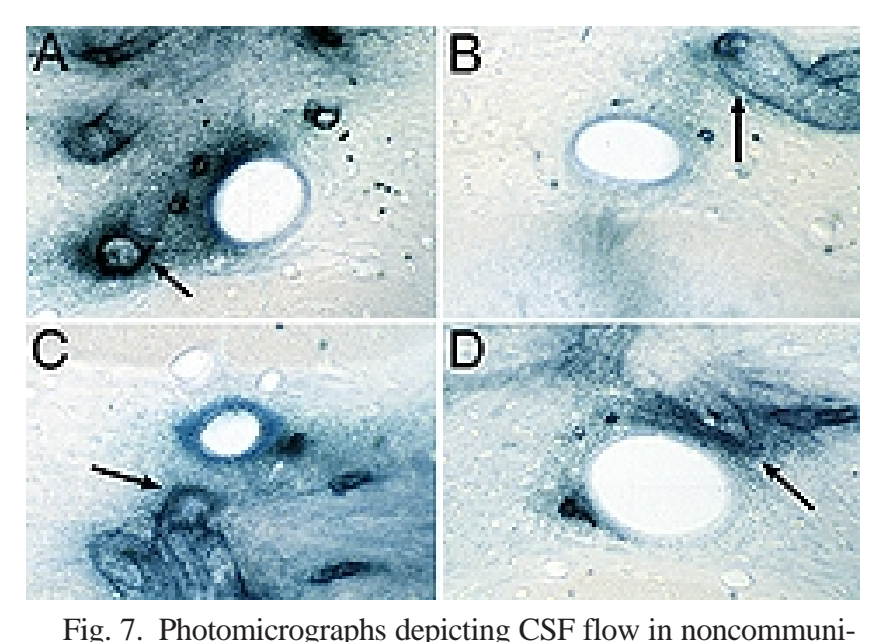

cating syringomyelia in transverse sections of cervical spinal cord. A: Cervical cord sample obtained 1 day after kaolin injection and 10 minutes after HRP injection into the cisterna magna. Horse(arrow) Horseradish peroxidase is present in perivascular spaces (arrow) as well as between the perivascular spaces and the central canal in a pattern indicating persistent CSF flow into the central canal. B: After 1 week postinjection the central canal is dilated, and there is evidence of CSF flow from perivascular spaces (arrow) to the central canal. C and D: At 6 weeks postinjection the central canal is dilated further, yet HRP is still present between perivascular spaces and the central canal in a pattern indicating a directed flow of CSF. Original magnification $\times 200$.

matic reduction of perivascular flow, and there was no evidence that CSF tracer reached the central grey matter perivascular spaces or central canal. The precise mechanism by which arterial pulsations cause a perivascular flow remains unclear. One possibility is that CSF is forced into the perivascular space from the subarachnoid space with pulsations transmitted from the head and large spinal arteries. A second possibility is that expansion of the penetrating arteries within the spinal cord forces fluid in the surrounding perivascular spaces preferentially toward the central grey matter and central canal.

\section{Fluid Flow in Noncommunicating Syringomyelia}

Our animal models of canalicular and extracanalicular syringomyelia are not perfect. Kaolin and QA each produces an inflammatory reaction, and it is possible that this reaction contributes to cyst formation. However, the presence of inflammatory cells should not dramatically influence the hydrodynamics of CSF flow, and the cysts produced with these models are remarkably similar pathologically to the human disease. Attempts are currently being made to produce an animal model of syringomyelia in which there is no inflammation.

The normal rapid perivascular-to-central canal flow of CSF continued at all stages of syrinx formation in the rat model of canalicular syringomyelia. Cerebrospinal fluid flowed from perivascular spaces preferentially into the central canal, even into segments of canal that had become isolated by occlusions and had dilated. The CSF also flowed into cysts in which there was evidence of pressure damage in the surrounding spinal cord. We were not able to obtain direct measurements of cyst pressure in rats. 


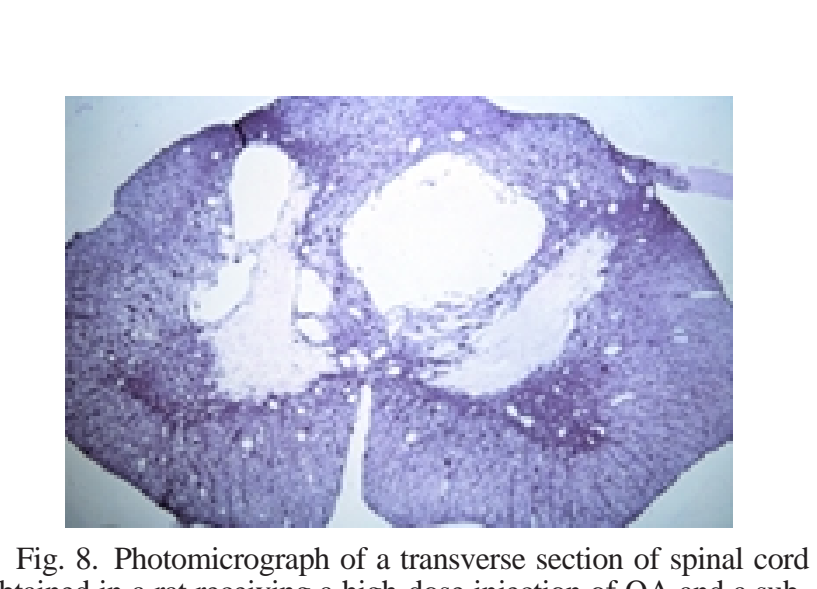

obtained in a rat receiving a high-dose injection of QA and a subarachnoid injection of kaolin. There are multiple large cysts in the dorsal half of the cord. $\mathrm{H} \& \mathrm{E}$, original magnification $\times 12$.

However, that the cysts enlarged and caused damage to the surrounding spinal cord suggests that the intracys pressure exceeded the spinal cord and subarachnoid space pressure at least at some stage of syrinx development. An arterial pulsation-driven perivascular flow of CSF from the subarachnoid space into isolated segments of central canal would explain the formation of cysts, their enlargement, and the development of pressures within the cyst that are higher than those in the surrounding spinal cord parenchyma.

In the model of extracanalicular syringomyelia an injection of an excitotoxic amino acid (QA) is used to produce focal neuronal injury and cell death. A cyst forms at the injection point and gradually enlarges. In our study larger cysts were produced using a higher concentration of QA Although production of subarachnoid arachnoiditis with kaolin was not sufficient to produce cysts, when arachnoiditis was produced in combination with the excitotoxic injury, multiple large cysts extending over many segments were created. It is possible that the mechanism by which the kaolin enhances cyst formation is related to the inflammatory reaction, although we believe that a mechanical effect on CSF flow is likely to be responsible. The kaolin-produced arachnoiditis results in a partial block of CSF flow in the subarachnoid space, which likely reduces the compliance of the subarachnoid space and thus increases the pulse pressure. An increase in pulse pressure might increase perivascular CSF flow. Although cysts formed using this model do not involve the central canal, our hypothesis is that perivascular flow is also responsible for the entry of CSF into the cysts as well as their enlargement. Before reaching the central canal, CSF flows from perivascular spaces into the interstitial space. Excitotoxic injury-produced cysts are essentially expansions of the extracellular space and could therefore receive the norma perivascular CSF flow.

\section{Clinical Implications}

Our general hypothesis proposes that canalicular syringomyelia occurs from arterial pulsation-driven perivascular CSF flow into an isolated segment of central canal and that extracanalicular cysts form initially as a result of injury (mechanical, hemorrhagic, or excitotoxic), with subsequent enlargement caused by active perivascular flow.
Cyst volume and pressure are a function of the balance between in- and outflow; formation and enlargement of a cyst, therefore, results from either an increase in inflow (increased perivascular flow) or a decrease of outflow (occlusion of the central canal or blockage of other outflow tracts). It is possible that a combination of factors is required to form a syrinx. For example, syringomyelia in association with Chiari malformation might require a combination of an isolated segment of central canal and an increase in subarachnoid space pulse pressure caused by the Chiari malformation. For posttraumatic syringomyelia to develop, a combination of an initial parenchymal cyst and an increase in subarachnoid space pulse pressure caused by arachnoiditis and reduced compliance may be required. This would explain why not every patient with a Chiari malformation or a spinal cord injury develops syringomyelia.

Many syringes resolve, or at least diminish in size, after posterior fossa decompressive surgery, lysis of arachnoid adhesions, or placement of a shunt. Placing a shunt in the subarachnoid space appears to be as effective as placing one in the syrinx. ${ }^{10,18}$ Some authors have claimed that posterior fossa decompressive surgery is effective because it relieves the valve effect of the cerebellar tonsils or permits normal flow of CSF across the foramen magnum. ${ }^{12,21} \mathrm{An}$ alternative explanation is that the decompressive procedure improves compliance, which reduces subarachnoid space pulse pressure and perivascular flow. Lysis of the adhesions and shunt placement could act by the same mechanism: improving compliance and changing the balance of syrinx fluid inflow and outflow in favor of outflow, thus collapsing the cyst. Changes in compliance caused by blockage of the shunt, reformation of arachnoid adhesions, and scarring at the foramen magnum could account for the relatively high recurrence rate associated with these treatments. If compliance is an important factor in recurrence rates, then a patch used to enlarge the dura at the foramen magnum or at areas of spinal arachnoiditis should be soft and flexible, not a material that promotes scar formation. Avoiding a patch or using relatively inert synthetic materials might prove more effective in the longer term than using autologous fibrous grafts such as fascia lata, which might be prone to cicatricial contraction. Investigation of the effects of compliance on perivascular fluid flow and syrinx formation will be a focus of our future research efforts.

\section{References}

1. Ball MJ, Dayan AD: Pathogenesis of syringomyelia. Lancet 2:799-801, 1972

2. Cifuentes M, Fernandez-LLebrez P, Perez J, et al: Distribution of intraventricularly injected horseradish peroxidase in cerebrospinal fluid compartments of the rat spinal cord. Cell Tissue Res 270:485-494, 1992

3. Cushing H: Studies on the cerebro-spinal fluid and its pathway. I. Introduction. J Med Res 26:1-19, 1914

4. Gardner WJ, Abdullah AF, McCormack LJ: The varying expressions of embryonal atresia of the fourth ventricle in adults. Arnold-Chiari malformation, Dandy-Walker syndrome, "arachnoid" cyst of the cerebellum, and syringomyelia. J Neurosurg

5. Heiss JD, Patronas N, DeVroom HL, et al: Elucidating the pathophysiology of syringomyelia. J Neurosurg 91:553-562, 1999 
Noncommunicating syringomyelia: experimental studies

6. Hinokuma K, Ohama E, Oyanagi K, et al: Syringomyelia. A neuropathological study of 18 autopsy cases. Acta Pathol Jpn 42:25-34, 1992

7. McLean DR, Miller JDR, Allen PBR, et al: Posttraumatic syringomyelia. J Neurosurg 39:485-492, 1973

8. Milhorat TH, Capocelli AL Jr, Anzil AP, et al: Pathological basis of spinal cord cavitation in syringomyelia: analysis of 105 autopsy cases. J Neurosurg 82:802-812, 1995

9. Milhorat TH, Johnson RW, Milhorat RH, et al: Clinicopathological correlations in syringomyelia using axial magnetic resonance imaging. Neurosurgery 37:206-213, 1995

10. Milhorat TH, Johnson WD, Miller JI: Syrinx shunt to posterior fossa cisterns (syringocisternostomy) for bypassing obstructions of upper cervical theca. J Neurosurg 77:871-874, 1992

11. Noguès MA: Syringomyelia and syringobulbia, in Vinken PJ, Bruyn GW, Klawans HL, et al (eds): Malformations. Handbook of Clinical Neurology, Vol 50. Amsterdam: Elsevier, 1987, pp 1-17

12. Oldfield EH, Muraszko K, Shawker TH, et al: Pathophysiology of syringomyelia associated with Chiari I malformation of the cerebellar tonsils. Implications for diagnosis and treatment. J Neurosurg 80:3-15, 1994

13. Peerless SJ, Durward QJ: Management of syringomyelia: a pathophysiological approach. Clin.Neurosurg 30:531-576, 1983

14. Rennels ML, Gregory TF, Blaumanis OR, et al: Evidence for a 'paravascular' fluid circulation in the mammalian central nervous system, provided by the rapid distribution of tracer protein throughout the brain from the subarachnoid space. Brain Res 326:47-63, 1985

15. Stoodley MA, Brown SA, Brown CJ, et al: Arterial pulsationdependent perivascular cerebrospinal fluid flow into the central canal in the sheep spinal cord. J Neurosurg 86:686-693, 1997

16. Stoodley MA, Gutschmidt B, Jones NR: Cerebrospinal fluid flow in an animal model of noncommunicating syringomyelia. Neurosurgery 44:1065-1076, 1999

17. Stoodley MA, Jones NR, Brown CJ: Evidence for rapid fluid flow from the subarachnoid space into the spinal cord central canal in the rat. Brain Res 707:155-164, 1996

18. Vassilouthis J, Papandreou A, Anagnostaras S, et al: Thecoperitoneal shunt for syringomyelia: report of three cases. Neurosurgery 33:324-328, 1993

19. Weed LH: Studies on cerebro-spinal fluid III. The pathways of escape from the subarachnoid spaces with particular reference to the arachnoid villi. J Med Res 31:51-91, 1914

20. Williams B: The distending force in the production of "communicating syringomyelia." Lancet 2:189-193, 1969

21. Williams B: On the pathogenesis of syringomyelia: a review. J R Soc Med 73:798-806, 1980

Manuscript received January 20, 2000

Accepted in final form February 1, 2000

Address reprint requests to: Marcus A. Stoodley, Ph.D., F.R.A.C.S, Institute of Neurological Sciences, Prince of Wales Hospital, Randwick, New South Wales 2031, Australia. email: m.stoodley@unsw.edu.au. 